\title{
Influence of the Driver Conditions on the Injury Outcome in Front Impact Collisions
}

\author{
Jacobo Antona ${ }^{1)}$ Susumu Ejima $^{2)}$ Yoshio Zama ${ }^{3)}$ \\ 1)-3) The Japan Automobile Research Institute \\ 2530 Karima,Tsukuba,Ibaraki,305-0822, Japan (E-mail: ajacobo@jari.or.jp)
}

Received on July, 5th, 2010

Presented at the JSAE Annual Congress on May, 21st, 2010

\begin{abstract}
Changes in the driver's posture and velocity caused by inertia during an emergency braking are believed to affect the injury outcome in front impact collisions. Moreover, at equivalent impact conditions, the results from real-world data analysis show the increased risk of injury in the cases in which an emergency braking preceded the accident. The objective of this study is to further investigate the relationship between such driver's pre-crash conditions and the injury outcome by using a computer human model. A finite element human model (JAMA model) and the results of the pre-impact experiments with volunteers were employed for this purpose. By simulating the same two accidents after different preimpact conditions it was concluded that the presence of a pre-impact emergency braking increased the severity of the loading mechanism sustained by the driver, especially on the thoracic region.
\end{abstract}

KEY WORDS: (Standardized) safety, finite element method (FEM), driver behavior (Free) emergency braking, injury mechanism, front impact, human model. [C1]

\section{INTRODUCTION}

Driver's evasive maneuver before the front impact collision is an important factor that may affect the injury outcome. The data of 860 front impact accidents occurred in Japan (19932004) were collected and analyzed by the Institute for Traffic Accident Research and Data Analysis (ITARDA) ${ }^{(1)}$. Two conclusions were extracted from this data analysis:

Firstly, it was concluded that the majority of the drivers made evasive maneuvers (braking and/or steering) before the impact.

Secondly, it was concluded that, with equivalent impact velocity, the occupant injury incidence rate was higher in the cases in which the driver braked just before the crash. This observation was especially significant in the analysis of chest injuries. The thoracic injury incidence ratio when the driver reacted with an emergency braking was higher than the cases in which the driver did not take any evasive maneuver

These conclusions are in accordance with other studies ${ }^{(2)}$ in which the analysis of the pre-crash reactions in real-world accidents was done to improve the understanding of the relationship between the pre-impact reactions and the injury outcome.

The apparent contradiction that relates braking with a possible higher injury risk can be explained. The braking reduces the impact velocity and hence the risk of injury. However, when cases with similar impact speed but different pre-impact driver actions are compared, the injury risk seems to be higher. The inertia of the occupant during the braking causes changes in posture, velocity, and relative position of the body in respect of the vehicle restraint systems. Such changes are hypothesized to affect the injury outcome. For example, the relationship between changes in posture and position and the injury output has been demonstrated both experimentally ${ }^{(3)}$ and with simulation work ${ }^{(4)}$. However, other phenomenon such as the influence of the inertial velocity of the occupant with respect to the restraint systems in the injury output has not been clarified yet.

This study is an effort to numerically replicate a frontal impact collision in order to better understand and quantify how the changes caused by the pre-impact driver reactions can affect the occupant kinematics and the possible injury outcome in a vehicle collision.

\section{NUMERICAL MODELS}

\subsection{Human Finite Element Model}

A human FE model developed and validated by the Japan Automobile Research Institute (JARI) and the Japan Automobile Manufacturers Association (JAMA) was employed in this research ${ }^{(5)}$. Figure 1 is a picture of the human model. It consists of a 50thpercentile male-based model that has been validated at length using a large amount of experimental data. Special effort was made in its thoracic region. 


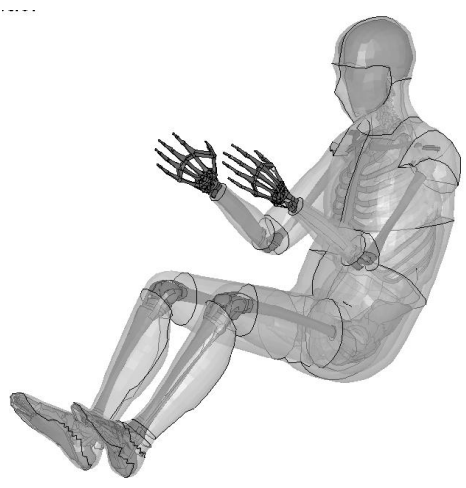

Fig 1. Human FE Model (JAMA model)

For the validation of the model, the results of component tests and table top tests with Post Mortem Human Subjects (PMHS) were used ${ }^{(6)}$. Moreover, the kinematics of the fullbody model was also validated against experimental data of the impact sled tests with full body PMHS ${ }^{(7)}$

\subsection{Vehicle Interior Finite Element Model}

The front half of a mid-sized vehicle interior model consisting of the main structure of the car, a seat, a 3-point seatbelt, the deformable column with an airbag, the instrument panel, and the floor panel was purchased from a software company (JSOL Corporation). Figure 2 illustrates the scheme of the vehicle interior model and the restraint systems.

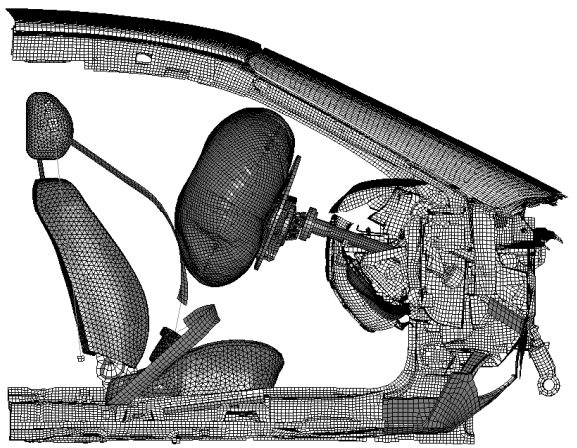

Fig 2. Vehicle Interior FE Model with restraint systems. (Some parts of the vehicle were deleted in this figure for visibility reasons)

The original model restraint systems were modified based on JARI's component experimental database. The seatbelt model includes a pyrotechnic retractor with a pre-tensioner. The retractor includes a force limiter $(4 \mathrm{kN})$. The pre-tensioner and the airbag deployment were programmed so that they trigger $10 \mathrm{~ms}$ and $20 \mathrm{~ms}$ respectively after the beginning of the vehicle impact.

\section{METHODOLOGY: NO BRAKING VS PRE-IMPACT BRAKING}

The main objective of this research was to understand how the reaction of the driver just before a front impact may affect the driver's kinematics and the injury outcome during the crash.
To achieve this goal, the two same frontal crashes after different pre-impact driver's reactions were numerically simulated and analyzed.

The first simulated scenario (named No braking case in this paper), represents a situation in which a vehicle running steadily at $55 \mathrm{~km} / \mathrm{h}$ sustains a frontal impact without any reaction of the driver. At the instant of the crash, the driver keeps an UMTRI position with no relative movement between the body and the vehicle.

The second simulated scenario (named Pre-impact braking case), represents an accident with the same impact velocity of $55 \mathrm{~km} / \mathrm{h}$. What differs from the previous case is the deceleration corresponding to an emergency braking just before the crash. Such deceleration provokes changes in the posture and in the relative velocity of the body in respect of the vehicle before the impact.

In both cases, the same crash pulse was used. It represents a 55 $\mathrm{km} / \mathrm{h}$ impact against a rigid wall with a $100 \%$ overlap. The deceleration curve is a half-sine wave with $28 \mathrm{G}$ at the peak and $100 \mathrm{~ms}$ duration.

The changes in human model's posture and velocity occurred due to inertial effects in an emergency braking phase were confirmed by using the experimental data with volunteers described in the following section.

\subsection{Pre-Impact braking experimental data}

Ejima et al. ${ }^{(8)}$ carried out experiments in which volunteers were subjected to decelerations equivalent to an emergency braking. The protocol used for such experiments was reviewed and approved by the Tsukuba University Ethics Committee, and the volunteers submitted their informed consent according to the Helsinki Declaration. For the experiments, a custom-designed sled to simulate frontal low-speed impacts was developed for this purpose. The volunteers were restrained with a lap belt and rigid foot plates. A three-dimensional motion capturing device (Eagle Digital Camera) was used to extract the subject's motion in an XY-Z space.
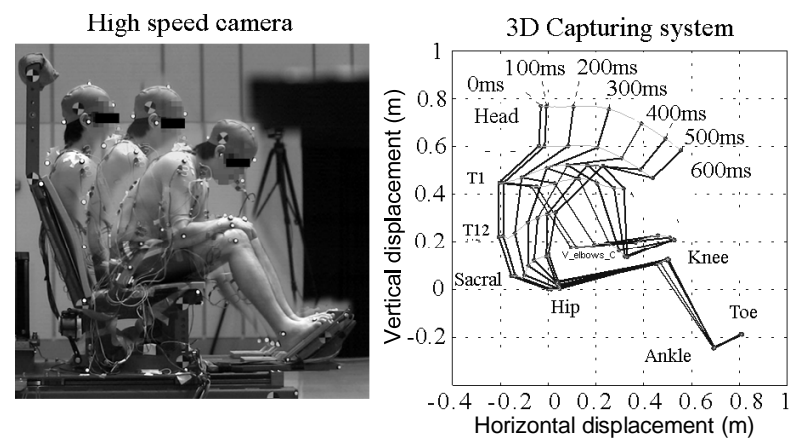

Fig 3. Physical motion of the volunteers captured with high speed camera (left) and with the 3D capturing system (right)

Figure 3 illustrates the scheme of the processed images captured with the high speed camera and the physical motions obtained with the 3D motion capturing system.

Within the tests carried out, the results corresponding with 5 males $(168-178 \mathrm{~cm}$ height and $62.4-75.6 \mathrm{Kg}$ weight) were selected and used to validate the human model in this specific research. In the selected cases, the subjects were asked to relax before the deceleration. The volunteers were unaware of the exact timing of 
the sled acceleration. The motion data obtained by the $3 \mathrm{D}$ capturing systems showed that during the first $200-300 \mathrm{~ms}$, the angles rotated by the first thoracic vertebra (T1) in respect of the hip ranged from 10 to 15 degrees, depending on the volunteer.

3.2. Validation of the human FE model motion during the emergency braking

The results of the sled tests with volunteers were utilized to validate the human FE model motion in the braking phase. The same environment with a rigid seat, foot plates, and a lap belt was reproduced. The $0.8 \mathrm{~g}$ pulse used for the experiments was prescribed to the numerical model and the calculated results were compared with the experimental data. Figure 4 shows superimposed pictures of the motion of the model at $0 \mathrm{~ms}, 200 \mathrm{~ms}$, and $300 \mathrm{~ms}$. At $200 \mathrm{~ms}$, the torso had rotated 15 degrees. The rotated angle of the torso was measured as the angle varied by the line passing by the sacrum and the first thoracic vertebra (T1).

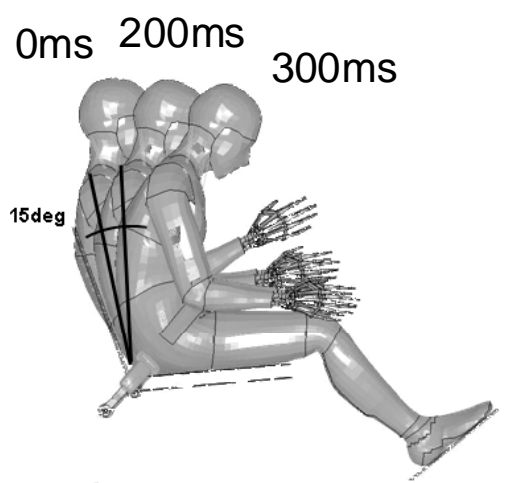

Fig 4. Physical motion of the human model

The calculated rotational velocity of the torso was also compared with the experimental results (Figure 5). The numerical results showed high correlation with the experimental measurements at the simulated $300 \mathrm{~ms}$

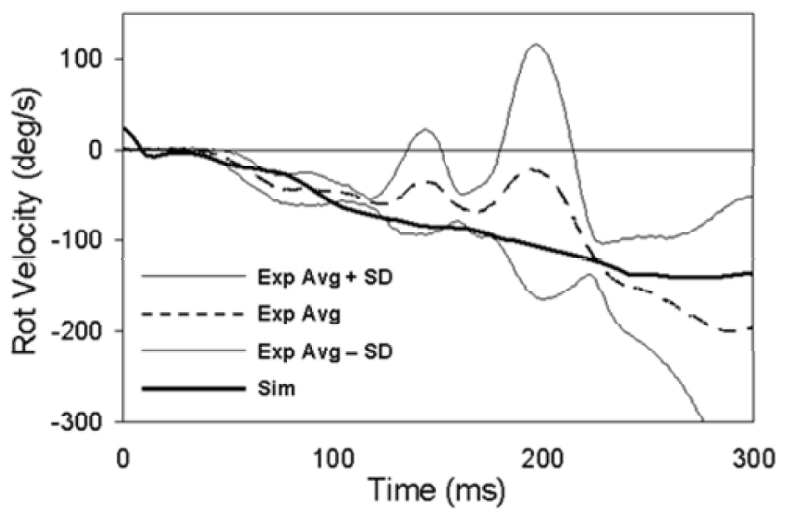

Fig 5. Comparison of experimental corridor (thin lines) and simulation results (thick continuous line) of torso $\mathrm{Y}$ rotational velocity (+Extension).

After the confirmation of the reasonable motion of the model during an emergency braking, the model was placed in the car interior model and the two scenarios under study were set and calculated as described in the next section.

\subsection{Simulation technique}

In the real world, humans are permanently under the effect of gravity. This can become an important factor when human FE models with many pre-stressed viscoelastic components such as the flesh, are used. However, to the knowledge of the author of this paper, there are no standard simulation procedures to account for this effect.

Another important issue was the absence of tools to accurately place human models with the desired position on the soft seat, while considering the deformation of the seat cushion and the flesh in contact with it

To account for these issues, a technique consisting on running the simulation in two phases was used. Such technique allows, first, to place the human model in the desired initial posture, and second, to re-initialize the pre-stressed soft parts (seat and flesh) to account for the effect of gravity from the beginning of the accident simulation.

In the first stage, the human model with an initial UMTRI position was placed just above the un-deformed seat cushion and allowed to be affected only by gravity. A long simulation was run, and it was observed that the body bouncing on the seat cushion greatly decreased after $300 \mathrm{~ms}$. During this phase, few nodes from the spine, head, arms and legs were constrained in longitudinal direction to keep the posture as close as possible to the UMTRI definition. Then, the geometry of the human model and the seat cushion deformed by gravity at $300 \mathrm{~ms}$ was copied into a different file. Using this geometry as a base, the seatbelt model was then developed so that it passed over the middle of the right clavicle and the center of the sternum.

The second stage corresponds with the actual accident simulation. The geometry of the occupant, and the restraint systems previously prepared was used. At this point, the ls-dyna (version 971R3) function Initial Foam Reference Geometry was applied to the soft tissues of the human model and to the seat foam. Such function allows pre-stressed soft materials in a different simulation to be re-initialized in an environment with different boundary conditions. Finally, the vehicle was prescribed with the vehicle deceleration pulse in the vehicle longitudinal direction.

For the No braking case, the $55 \mathrm{~km} / \mathrm{h}$ crash pulse was input into the vehicle frame, and a simulation of $120 \mathrm{~ms}$ was run. For the Pre-impact braking case, a $320 \mathrm{~ms}$ acceleration pulse was generated. The first $200 \mathrm{~ms}$ of the curve were obtained from the emergency braking sled experiments previously described $(0.8 \mathrm{G})$. The second part of the curve was the same $55 \mathrm{~km} / \mathrm{h}$ crash pulse.

This technique, allows to account for the stress of soft parts caused by gravity, and to have an accurate control of the initial posture of the driver and the design of the seatbelt.

\section{RESULTS}

\subsection{Kinematics}

Figures 6 and 7 show a sequence of lateral and top views of the simulated occupant's physical motion during the collision for the No braking case and the Pre-Impact braking case, respectively.

In the No braking case, little motion forward of the occupant occurs at the first $40 \mathrm{~ms}$. The airbag deploys completely before it 
contacts the body. While the face and the chest contact the airbag, the hip pushes the cushion down and forward, causing its deformation. At $70 \mathrm{~ms}$, the hip and the knees reach their maximum traveling distance constrained mainly by the lap belt and the seat cushion. Meanwhile, the upper torso keeps on moving forward until $90 \mathrm{~ms}$. During the lapse from 90 to $100 \mathrm{~ms}$, the shoulders and the head reach their maximum forward displacement. From then on, the rebound phase starting with the hip and propagating to the back and the head occurs. In this phase, the whole body moves backwards and separates from the airbag.

In the Pre-Impact braking case, the movement of the upper torso can be observed from the beginning of the impact. The face and the chest contact the airbag before its complete deployment. While the airbag keeps on inflating and wraps around the upper chest-neck-head complex, the whole body moves forward and downward against the seat cushion, causing its deformation. At $70 \mathrm{~ms}$, the hip and the head reach their maximum forward traveling distance, stay there for a few ms, and initiate their rebound phase. While this happens, the chest and the shoulders keep on moving forward up to $90 \mathrm{~ms}$. The right shoulder moves slightly due to the constraint by the shoulder belt, and the inflated airbag, while the free left shoulder moves forward faster. This causes a rotation to the right of the whole upper torso. Such rotation strongly affects the body kinematics for the rest of the crash. Finally, from $90 \mathrm{~ms}$ the rebound of the rotated thorax starts, and the whole body rebounds back and leftwards.

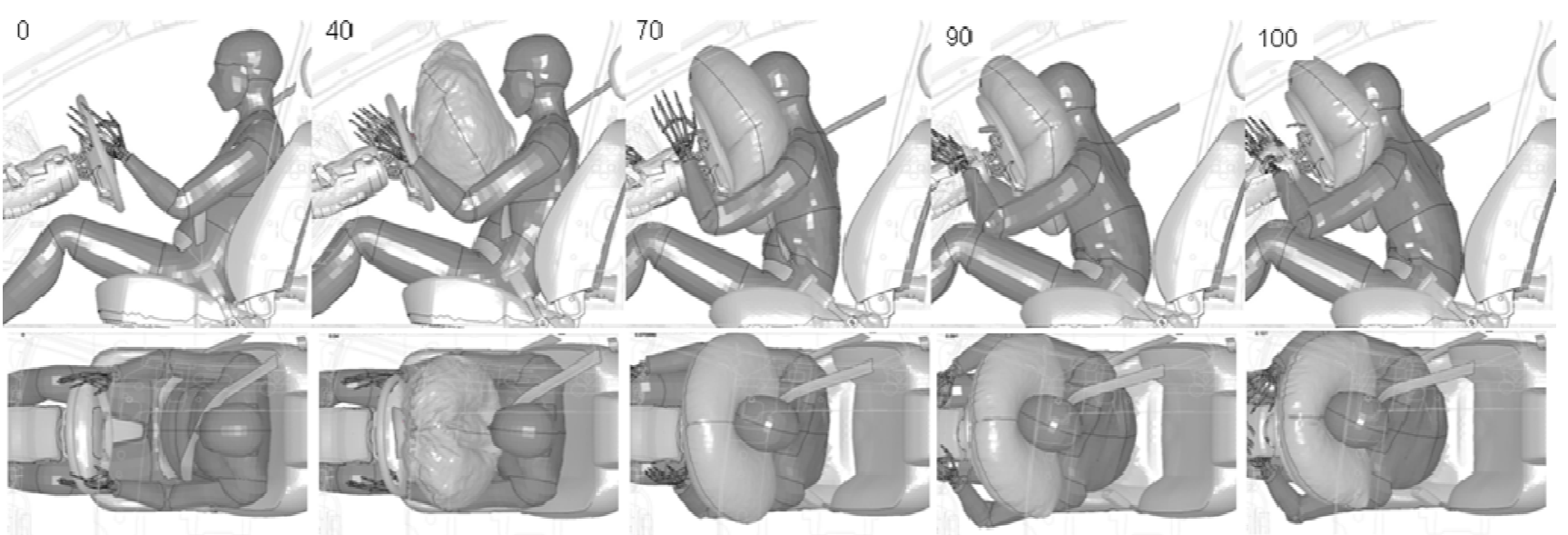

Fig 6. Occupant's kinematics during the crash in the No braking case at $0,40,70,90$, and $100 \mathrm{~ms}$

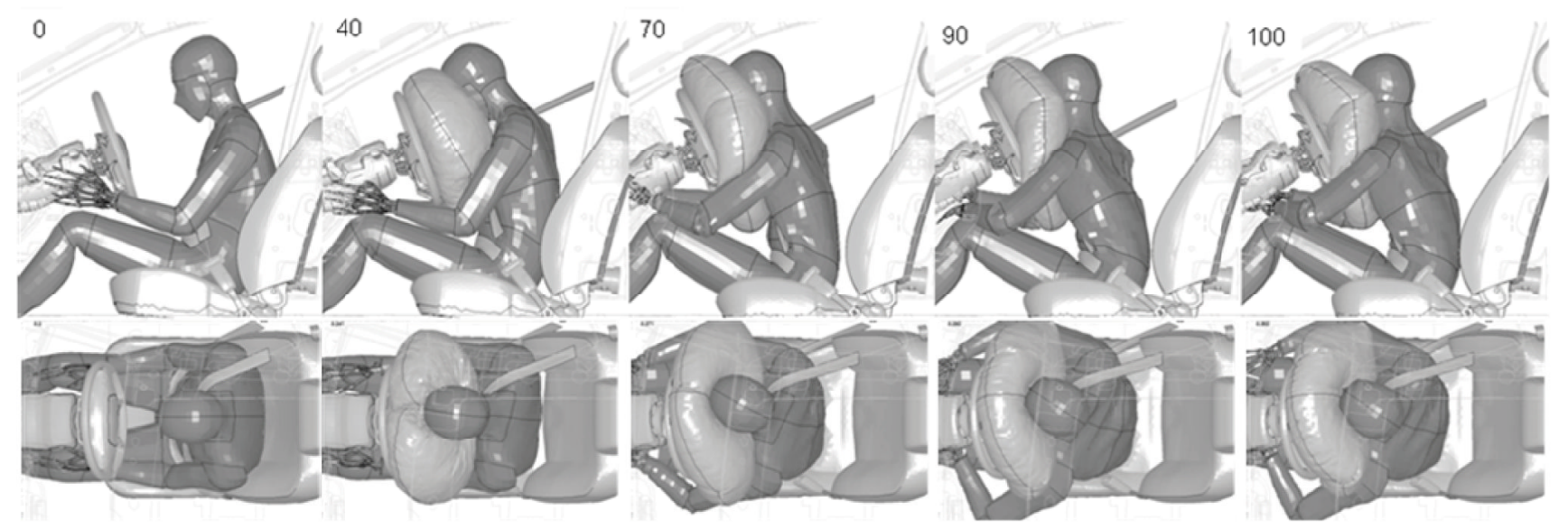

Fig 7. Occupant's kinematics during the crash in the Pre-impact braking case at $0,40,70,90$, and $100 \mathrm{~ms}$.

\subsection{Shoulder Belt forces}

Figure 8 shows a comparison of the shoulder belt forces of the two cases under study. Important differences were detected in the initial peak force caused by the pre-tensioner, in the shape of the loading curve and in the maximum plateau force.

During the pre-tensioning in the No braking case, the seatbelt is reeled-in by approximately $70 \mathrm{~mm}$ into the retractor. In this phase the load of the shoulder belt rapidly rises from 0 to $1800 \mathrm{~N}$. The retractor locks at $27 \mathrm{~ms}$, and the belt load increases from then on in two phases. In the first phase, the belt load reaches $5000 \mathrm{~N}$ at $43 \mathrm{~ms}$, and at the smoother phase it keeps on increasing until it reaches a plateau force of $6000 \mathrm{~N}$ at approximately $70 \mathrm{~ms}$. The plateau force continues until $95 \mathrm{~ms}$, at which the belt unloading occurs.

In the Pre-impact braking case, the belt is reeled-in into the retractor by about $45 \mathrm{~mm}$ causing a sudden rise of the shoulder belt load to $2400 \mathrm{~N}$. The retractor locks at $24 \mathrm{~ms}$, and from then on the load rises constantly and reaches a plateau force of $6700 \mathrm{~N}$ at $40 \mathrm{~ms}$. 
The load remains almost constant until 90ms, when the unloading begins.

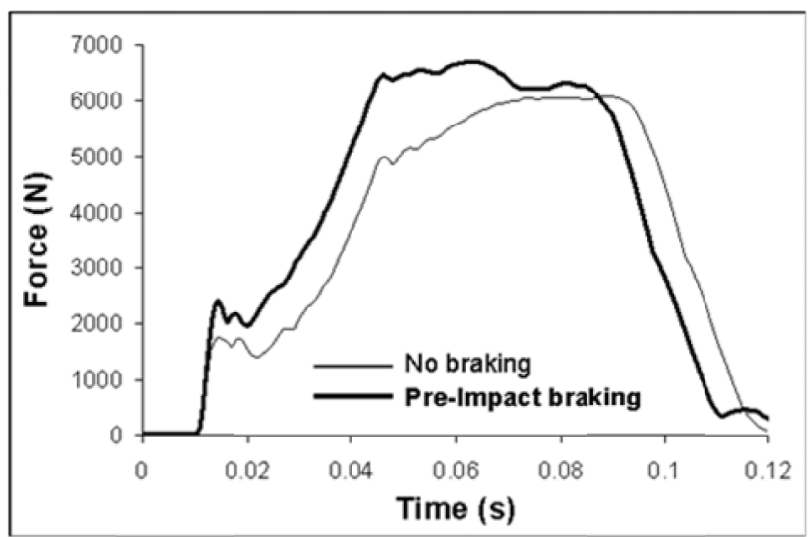

Fig 8. Shoulder belt forces during the impact.

\subsection{Injury Indicators and injury risk}

Due to the presence of the airbag, the head did not impact any stiff part of the vehicle. HIC36 values of 223 and 194 were obtained in the non-reaction case and the emergency braking case, respectively. Such values are much lower than the injury threshold for humans. Thus no further discussion with regard to potential head injuries is made in this paper.

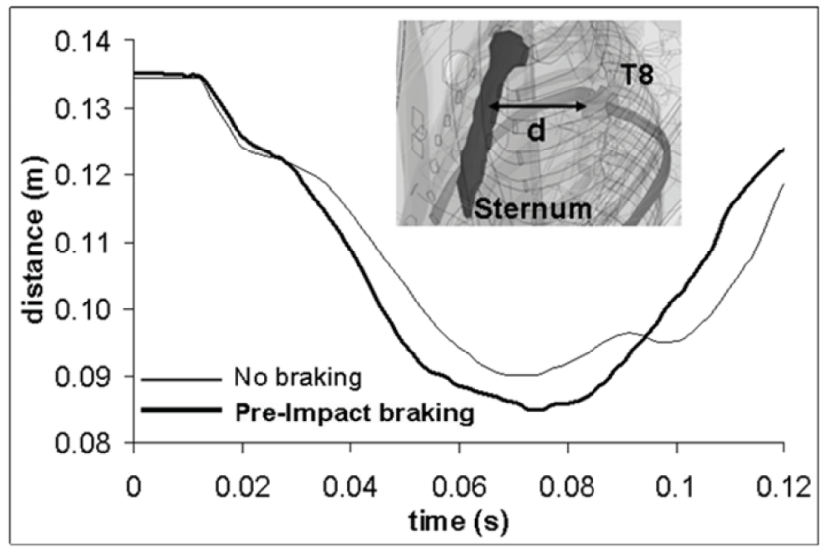

Fig 9. Chest deformation during the impact, measured as the absolute distance between the eighth thoracic vertebra and the center of the sternum.

No important differences were identified when chest accelerations were compared. However, significant differences were detected in the chest compression. Figure 9 shows the measured chest deformation in both cases.

From $0 \mathrm{~ms}$ to $30 \mathrm{~ms}$ both cases follow a similar path. However, from $30 \mathrm{~ms}$, the chest deformation in the Pre-impact braking case tends to deviate from the No braking case, reaching the higher maximum value. A maximum chest deformation of $44 \mathrm{~mm}(33 \%)$ was observed in the No braking case, while the scenario including the driver's pre-crash reaction resulted in a maximum deformation of $51 \mathrm{~mm}(38 \%)$. Kroell et al. ${ }^{(9)}$ concluded that the maximum thorax compression is well correlated with the Abbreviated Injury Scale (AIS) and proposed the Compression Criterion. Statistical analysis of the injury risk showed that in frontal impact, a thorax compression of $35 \%$ results in a $25 \%$ probability of severe injuries AIS4 or higher.

Another important phenomenon observed that could lead to an increased risk of injury was the torsion sustained by the neck. Figure 10 shows the obtained neck torsion angle for both cases under study. It was identified at the late stage of the contact with the airbag for both cases, showing higher rotation angles for the Pre-Impact braking case.

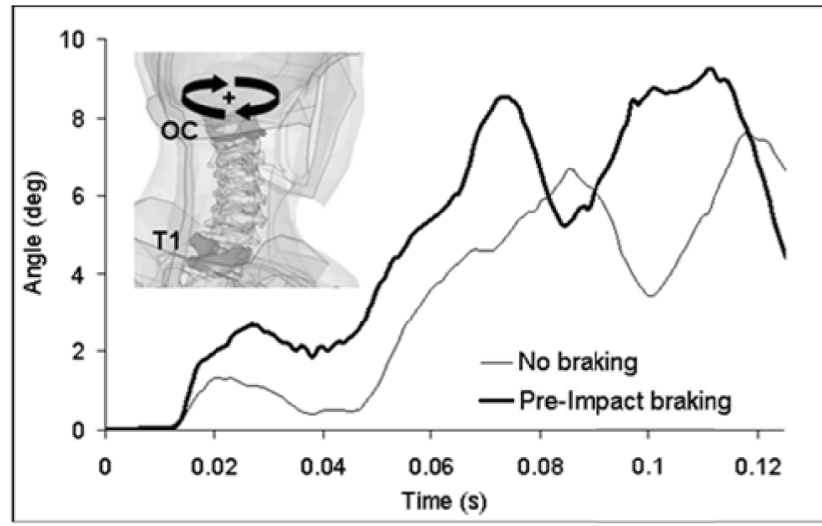

Fig 10. Neck torsion during the impact, measured as the angle rotated by the Occipital Condyle with respect to the first thoracic vertebra

\section{DISCUSSION}

Changes in posture and body velocity caused by inertia during the emergency braking resulted in important differences in body kinematics, occupant loading patterns, and injury indicators during the collision.

In the No braking case, the upper torso was initially leaning back against the seatback. The pre-tension contributes to keep the torso far from the steering column. This allowed the airbag to completely unfold before it contacted the occupant. The inflated airbag in combination with the shoulder belt delivered a considerable amount of energy to the thorax, provoking its deformation. The chest reached a maximum compression of $44 \mathrm{~mm}$ $(33 \%)$.

In the pre-impact braking case, when the crash began, the occupant torso was leaning forward (=Flexion) by approximately 15 degrees and moving with a rotational velocity of $135 \mathrm{deg} / \mathrm{s}$. On the one hand, the initial leaning-forward position of the occupant in combination with the initial kinetic energy of the driver led to a higher belt pretension peak force and a higher belt plateau than in the previous case. On the other hand, the driver's initial out-ofposition by itself strongly affected the upper body-airbag interaction and hence the chest-neck loading mechanism. Horsch et al ${ }^{(10)}$ carried out experiments with out-of-position dummies and explained the chest-neck airbag membrane loading mechanism, which occurs in the late airbag deployment process when the occupant is too close to the airbag system. The airbag wraps around the upper chest and the neck of the occupant, and it produces strong force over a large area ${ }^{(11)}$. Lebarbe et al. (2005) conducted 
experiments with PMHS to better understand the thoracic injuries generated by the airbag membrane loading effect. Although these authors made important advances in the understanding of the interaction between out-of-position occupant and the airbag, they did not mention the possibility of having combined neck extension and rotation as a result of the asymmetric displacement forward of the torso before and during its interaction with the airbag loading.

In the simulations implemented in this research, the membrane loading phenomenon was clearly identified only when the emergency braking was taken into consideration. Such effect is an important factor contributing to a bigger chest compression. Such an airbag loading mechanism in combination with a severer shoulder belt loading resulted in a maximum chest deformation of $51 \mathrm{~mm}(38 \%)$.

\section{LIMITATION OF THE STUDY}

The muscular activity of the humans involved in car crashes is an important factor that may affect to the kinematics at the braking phase and to the injury output in several ways.

For this specific work, the motion of the model in the emergency braking phase was verified against sled test data with relaxed volunteers. In this case it was assumed that the muscle activity of the volunteers did not affect the kinematics of the torso for at least $200 \mathrm{~ms}$. Seen the results, such assumption can be considered correct for the validation of the model.

However, in real-life accidents, the influence of reactions such as bracing while holding the steering wheel, or interposing the upper extremities between the airbag and the body as a protective reflex, could become an important factor affecting the injury output. Those kinds of reactions are supported by reflexive muscular reaction.

Since neither reflexive reaction, nor muscle activity were considered to simulate such kind of pre-impact reactions, the results and conclusions from this research should be understood within the context of the conditions previously described in this report, and under the limitations addressed here.

\section{CONCLUSIONS}

A $55 \mathrm{~km} / \mathrm{h}$ frontal impact accident was numerically simulated by using the FE model. A validated human model and a vehicle interior model were employed for this purpose. Two different pre-impact driver reactions were simulated, and the influence of such reactions on the kinematics, the body loading patterns, and the possible injury outcome was analyzed.

In the first case, which simulated the scenario in which the driver does not take any evasive maneuver before the accident, the driver had an UMTRI position at the moment of the impact. A pretension peak force of $1800 \mathrm{~N}$ and a shoulder belt plateau force of $6000 \mathrm{~N}$ were confirmed in this case. The airbag completely opened before contacting the occupant model. Throughout the collision, the occupant's head, chest, and shoulders moved forward in relatively parallel paths and reaching their maximum traveling distance almost simultaneously. The energy delivered by the airbag and the shoulder belt to the upper torso caused a maximum chest deformation of $44 \mathrm{~mm} \mathrm{(33 \% ).}$

The second case simulates a scenario in which the driver reacts with an emergency braking just before the crash. In the braking phase, due to inertia, both the driver's posture and initial velocity change. The occupant's initial posture and velocity lead to higher shoulder belt pretension peak force and plateau force of $6700 \mathrm{~N}$. Such belt forces cause the rotation of the torso to the right before and during the contact with the airbag. The out-of-position also provokes the early contact with the airbag, leading to membrane loading mechanism. This results in the increase of the pressure on the thorax. The belt force in combination with the loads delivered by the airbag into the torso caused a maximum chest deformation of $51 \mathrm{~mm}(38 \%)$.

It is concluded based on these results that, at the equivalent impact speed of $55 \mathrm{~km} / \mathrm{h}$, the changes in posture and velocity of the driver caused by inertia during an emergency braking affect the kinematics and the injury output during the crash. Moreover, higher belt load, torso traveling forward distance, chest compression, and neck rotation were observed when the emergency braking maneuver was taken before the crash.

The authors of this report believe in the importance of taking into consideration the driver's reaction prior to a front impact crash for future safety regulations and for developing safer restraint system.

\section{REFERENCES}

(1) Accident Analysis Report (JAPAN). Institute for Traffic Accident Research and Data Analysis (ITARDA), 2007.

(2) Thomas, C., Hermitte, T., Perron, T., Le Coz, J.: Driver Actions during Real-World Pre-crash Phases. JSAE 1999 Proceedings 992520 .

(3) Lebarbe, M., et al.: Thoracic Injury Investigation using PMHS in Frontal Airbag Out-of-Position Situations. Stapp Car Crash Journal, Vol.49, 2005.

(4) Bose, D, Crandall, JR, Untaroiu, CD, Maslen, E.: Influence of pre-collision occupant properties on the injury response during frontal collision. IRCOBI Conf., 2008.

(5) Zama, Y., et al.: Development of finite element human model for assessment of injuries in front impact, JSAE 2010.

(6) Kent, R., et al.: Frontal thoracic response to dynamic loading: the role of superficial tissues, viscera, and the rib cage, IRCOBI Conf., 2005.

(7) Show, G.,et al.: Frontal impact PMHS sled tests for FE torso model development, Proc. of IRCOBI Conf., 2009.

(8) Ejima, S., et al.: Prediction of Pre-Impact occupant kinematic behavior based on the muscle activity during frontal collision, $21 \mathrm{st}$ ESV, 2009.

(9) Kroell, et al.: Impact tolerance and response to the human thorax, Proc. 15th Stapp Car Crash Conf., 1971.

(10) Horsch, J., et al.: Assessment of airbag deployment loads. 34th Stapp Car Crash Journal, 1990.

(11) Schmitt, K.U., et al.: Trauma Biomechanics. Introduction to Accidental Injury. Springer 